\title{
La evolución de la enseñanza quirúrgica: un desafío permanente
}

\author{
The evolution of surgical teaching: a permanent challenge
}

Jorge Martínez C. ${ }^{1}$

El tímido inicio de la cirugía en Chile se remonta al siglo XIX con las contribuciones de cirujanos chilenos, como el Dr. Pedro Morán (1771-1840) y de notables profesores extranjeros como los Drs. Nataniel M. Cox (1785-1869), inglés, y Lorenzo Sazié (1807-1865), francés. También de destacados discípulos de aquéllos, como el Dr. José Joaquín Aguirre (1822-1901), entre otros ${ }^{1}$.

Así, la formación de cirujanos tiene una larga tradición en diversos hospitales, tanto en nuestra capital, como también en establecimientos de regiones, siguiendo el modelo "maestro-aprendiz" iniciado por Williams Halsted en 1889 en Norteamérica ${ }^{2}$. Este modelo tutorial y jerarquizado, que formó a un sinnúmero de cirujanos en todo el mundo, se basó en el aprendizaje progresivo a partir de la figura de un tutor a quien se observa, luego se imita y finalmente, el discípulo desarrolla una autonomía progresiva, que lleva al cirujano a repetir el modelo en el rol de profesor. Esto siempre en base a un gran número de pacientes, que se entregaban dócilmente a la práctica de la cirugía, haciendo plena confianza de sus cirujanos. Sin embargo, hoy -frente a los crecientes y rápidos desarrollos tecnológicos, al incremento en la especialización de varias áreas de la cirugía y al actual requerimiento de altos estándares de calidad y seguridad en la atención del paciente -este modelo clásico tiende a desfallecer ${ }^{3}$. Es por esto, que la mayor parte de las residencias de cirugía general han tenido que adaptar sus métodos de enseñanza quirúrgica.

La laparoscopía para cirujanos se introdujo inicialmente para procedimientos simples: laparoscopía diagnóstica, apendicectomía y colecistectomía, lo que constituyó la revolución de la cirugía del pasado siglo XX. Con el tiempo, su aplicación se fue extendiendo a escenarios más complejos. En las primeras etapas, la enseñanza de las habilidades laparoscópicas en residencias de cirugía general, fue difícil y no estandarizada, considerando la propia curva de aprendizaje de los tutores, y que el aprendizaje de aquellas exigía el desarrollo de habilidades motoras y espaciales que eran y aún son más complicadas de adquirir ${ }^{4}$. Para enfrentar estos desafíos, los programas de residencia de cirugía general han incorporado cambios teniendo en cuenta los siguientes elementos: enseñar mediante la práctica bajo supervisión; entrenamiento paso a paso de complejidad creciente; un entorno de enseñanza más cercano entre el cuerpo docente y los residentes; tutoría generosa por parte de los profesores cirujanos; y, más recientemente, programas estructurados de simulación en laparoscopía básica y avanzada. Esto ha coincidido, en nuestra experiencia, con un incremento de los procedimientos laparoscópicos realizados bajo supervisión por los residentes, con un progresivo incremento en operaciones laparoscópicas más complejas, como sutura de úlceras perforadas, hernias y procedimientos bariátricos. En varios programas de residencia quirúrgica, la simulación ha desempeñado un papel crucial en la adquisición de habilidades quirúrgicas con transferencia de estas habilidades al quirófano ${ }^{5}$. Por otra parte, ha permitido acortar las curvas de aprendizaje en la adquisición de habilidades laparoscópicas. Conscientes de estos cambios, la Sociedad de Cirujanos de Chile (SCCH) y su Departamento de Educación están desarrollando un ambicioso proyecto para implementar Centros de Simulación Quirúrgica en diversas regiones del país, para así facilitar este aprendizaje a nuestros cirujanos que ejercen alejados de los centros universitarios de Santiago.

Este desarrollo exponencial de la cirugía mínimamente invasiva ha dejado necesariamente de lado, los espacios de tiempo para la adquisición de las habilidades de la cirugía clásica con apertura espaciosa de cavidades. Así, recientes encuestas a egresados de diversos programas universitarios han señalado una deficiencia en la adquisición de destrezas y habilidades para enfrentar el trauma y urgencias
'Profesor Asociado. Jefe de Programa Residencia de Cirugía General, Pontificia Universidad Católica de Chile.

Correspondencia a: Jorge Martínez C. jamartin@med.puc.cl 
vasculares (datos no publicados). Nuevamente en esta carencia, puede haber una oportunidad: crear modelos simulados ${ }^{6}$ que permitan durante las residencias adquirir competencias básicas y avanzadas para el adecuado enfrentamiento de una patología que será cada vez más prevalente y que la formación de subespecialistas está lejos de poder dar abasto ${ }^{7,8}$. En este sentido, la apertura de rotaciones en regiones a través del Departamento de Educación de la $\mathrm{SCCH}$ permite contribuir a una mayor exposición de los residentes a operaciones de diversa complejidad y a su capacitación mas integral.

Finalmente, quisiera desde esta tribuna llamar a la reflexión, si no es acaso el momento, de que los programas de residencia de cirugía general se extiendan a un período de 4 años, debidamente y progresivamente mejor financiados, para otorgar al país un cirujano más maduro, competente y autónomo, líder frente a los desafíos de nuestra patología prevalente.

\section{Bibliografía}

1. Cubillos L. Historia de la Sociedad de Cirujanos de Chile. Primera parte. Rev Chil Cir. 2012;64:312-8.

2. Cameron JL. William Stewart Halsted: Our surgical heritage. Ann Surg. 1997;225:445-58

3. Richard K.Reznick HM. Changes in the wind. N Engl J Med. 2006;355:2664-9.

4. Grantcharov TP, Funch-Jensen P. Can everyone achieve proficiency with the laparoscopic technique? Learning curve patterns in technical skills acquisition. Am J Surg. 2009;197:447-9.

5. Boza C, Leon F, Buckel E. Simulationtrained junior residents perform better than general surgeons on advanced laparoscopic cases. Surg Endosc. 2017;31:135-41.

6. Sutherland LM, Middleton PF, Anthony A, Hamdorf J, Cregan P, Scott D, et al.
Surgical simulation: a systematic review. Ann Surg. 2006;243:291-300.

7. Bowyer MW, Kuhls DA, Haskin D, Sallee RA, Henry SM, Garcia GD, et al. Advanced Surgical Skills for Exposure in Trauma (ASSET): the first 25 courses. J Surg Res. 2013; 183: 553-8.

8. Cherry RA, Ali J. Current Concepts in Simulation-Based Trauma Education. J Trauma Acute Care Surg. 2008;65:118693. 